\title{
DOKUMENTATION
}

\section{Staatsangehörigkeitsbestimmungen in den Verfassungen der Welt}

Von Hellmuth Hecker

\begin{abstract}
Als Herbert Krüger am 1. 4. 1955 die Leitung der Forschungsstelle für Völkerrecht und ausländisches öffentliches Recht und des Instituts für Auswärtige Politik übernahm, war die Idee von Eberhard Menzel, ein Verfassungsregister aller Gebiete der Welt zu erstellen, erst eben in einem Band (Deutschland) verwirklicht worden. Unter dem Direktorat von Krüger konnte im nächsten Jahre der Band Europa erscheinen (1956) sowie bald auch der Band Amerika (1958). Den Abschluß bildete am Ende der ersten Entkolonialisierungswelle der Band Afrika-Asien-Australien (1963), der wegen schwierigen Finanzierungsfragen nur durch den Einsatz Krügers erscheinen konnte.

Um dieses umfangreiche und unentbehrliche Registerwerk fortzuschreiben und die neuesten Entwicklungen, insb. die weiteren Verselbständigungen von Gebieten in allen 5 Kontinenten zu berücksichtigen, wurde der Weg gewählt, in dieser Zeitschrift darüber zu berichten. So erschienen hier folgende Beiträge:
\end{abstract}

M.I. Kehden: Lateinamerika, VRU 1968, S. 196-224

B.-O. Bryde/H. Hecker: Afrika 1975, S. 233-282

Hecker: Asien/Australien 1976, S. 81-113, 241-278

Hecker: Amerika 1979, S. 187-215, 299-317, 433-455

Der hier folgende Beitrag enthält eine Aktualisierung der bisherigen Registerbände, indem er die Verfassungen aller 175 Staaten der Welt und von 18 abhängigen Gebieten, die eine Staatsangehörigkeitsbestimmung haben, enthält. Die Reihenfolge der Kontinente entspricht dem Titel dieser Zeitschrift: Afrika, Asien (mit Australien), Amerika. Zwecks allgemeiner Rechtsvergleichung beschränkt sich die Ubersicht nicht nur auf die Entwicklungsländer, sondern führt auch die Industrienationen Nordamerikas (USA, Kanada) und Europas mit auf. Dabei werden alle Staaten berücksichtigt, die effektive Staatsgewalt ausüben, unabhängig von ihrer Anerkennung durch die Völkerrechtsgemeinschaft. Am Schluß folgen StA-Vorschriften in Verfassungen abhängiger Gebiete von 9 "Kolonialstaaten". Nach der Anzahl der Staaten zeigen die fünf Kontinente folgendes Bild:

Afrika: 55 Staaten

Asien: 38 Staaten

Europa: 36 Staaten

Amerika: 35 Staaten

Australien: 11 Staaten 
Ohne eine Verfassung im formellen Sinn (einheitliches Dokument) leben 9 Staaten. Von ihnen haben 3 früher eine Verfassung gehabt; sie ist aber aufgehoben worden: Äthiopien, Burkina-Faso, Laos. 6 Staaten haben bisher noch nie eine geschriebene Verfassung besessen; nämlich in Europa: Andorra, Großbritannien und San Marino; in Asien: Israel, Oman und Saudiarabien. Lediglich zeitweise suspendierte Verfassungen sind noch aufgeführt. Nicht nur die Bundesrepublik Deutschland sondern auch eine Reihe von Staaten bezeichnen ihre Verfassung als Grundgesetz (bzw. -statut, -akt, -prinzipien), und zwar in Europa: Dänemark, die Niederlande, Norwegen, der Vatikan; in Amerika: Guatemala und Surinam; in Afrika: Äquatorialguinea, Benin, Tschad; und in Asien: Afghanistan.

Die Namen der jeweiligen Verfassungen werden bei germano-romanischen Sprachen im Original angegeben, sonst in deutscher Ubersetzung. Das Datum in Klammern ist das des Inkrafttretens, soweit dies feststellbar war. Ein Gleichheitszeichen bedeutet, daß beide Daten übereinstimmen. Die älteste Verfassung der Welt, die heute noch gilt, ist die der USA (1787), es folgen Norwegen und die Niederlande (1815). Formell sind diese beiden Verfassungen trotz unzähliger Änderungen und Umnumerierungen noch als identisch anzusehen.

Von den 175 Staaten haben nur 45 keinerlei Regelung der Staatsangehörigkeit, während $3 / 4$ aller Staaten irgendeire solche Vorschrift kennen. Das zeigt deutlich, wie die DreiElementen-Lehre des Staates noch heute wirksam ist. Die 45 Staaten ohne eine StA-Regelung verteilen sich wie folgt auf die Kontinente:

Europa: $\quad$ Polen, Rumänien, Ungarn, Vatikan

Asien: $\quad$ Bhutan, Japan, Kamboja, Nord-Korea, Thailand

Australien: $\quad$ Neuseeland, Westsamoa

Afrika: Algerien, Angola, Benin, Djibouti, Elfenbeinküste, Gabun, Guinea, Guinea-Bissau, Kamerun, Kongo, Lesotho, Libyen, Madagaskar, Mali, Marokko, Mauretanien, Mozambik, Niger, S. Tomé, Südafrika, Swaziland, Togo, Tschad, Zentralafrika.

Es fällt auf, daß knapp die Hälfte der Staaten Afrikas (24 von 55) keine Staatsangehörigkeitsregelung kennt. Es sind hier: vor allem ehemals französische Staaten (16), alle ehemals portugiesischen und nur 2 ehem. englische Staaten (Südafrika, Swaziland) sowie das ehem. italienische Libyen. Dabei waren es die französischen Revolutionsverfassungen von 1791-1799, die das materielle StAR erstmals in einer Verfassung regelten.

Der Inhalt der StAB'en läßt sich in formelle und materielle Bestimmungen differenzieren, wobei als letztere nur Erwerbs- und Verlustgründe gelten sollen. 


\section{Formelles Recht}

\section{1) Verweisung auf Gesetze}

Die minimalste StAB in einer Verfassung verweist für die Regelung (Erwerbs- und Verlustgründe) auf die Gesetze. Diese materiellen Vorschriften dürfen dann nur durch Gesetz und auf Grund eines Gesetzes erlassen werden.

Ein entsprechender Gesetzesvorbehalt findet sich bei sehr vielen Staaten:

Afrika:

Ägypten, Bophutatswana, Burundi, Malawi, Rwanda, Senegal (als Zuständigkeitskatalog), Somalia, Sudan, Tunis, Zaire.

Asien: Afghanistan, Bangladesh, Bahrain, Burma, Indonesien, Irak, Jordanien, Korea (Süd), Kuwait, Libanon, Qatar, Syrien, Vereinigte Arab. Emirate, Yemen (Nord)

Amerika:

Surinam

Europa:

Albanien, Belgien (verweist auf C.c.), Bulgarien, DDR, Frankreich, Irland, Liechtenstein, Monaco (mit weiteren), Portugal, Schweden, Schweiz (m. weiteren), Spanien, Sowjetunion, Zypern (Nord), Türkei (m. weiteren)

\section{2) Bundesstaatsklauseln}

In allen Bundesstaaten (außer Südafrika) ist bestimmt, daß der Bund für die Regelung der Bundes-StA zuständig ist, d. h. für die StA im völkerrechtlichen Verkehr. Eine Zuständigkeit der Länder für eine ev. Regelung der Länder-StA sehen nur die Bundesrepublik, Osterreich, die Schweiz und die CSSR vor. Die CSSR regelt sogar nur die Grundsätze der StA, während die Einzelheiten den StAG'en der beiden Länder (Tschechei u. Slowakei) überlassen sind. Ferner ist jeder Bürger der beiden Länder dadurch Bürger der CSSR, ebenso wie in der Schweiz jeder Kantonsbürger dadurch Schweizerbürger ist.

In Jugoslawien haben die 6 Republiken 1974-1979 eigene StAG'en erlassen. Ebenso haben 25 Schweizer Kantone (außer Jura) eigene StAG'e (Bürgerrechtsgesetze), das älteste 1840 (Wallis), das neueste 1978 (Zürich). Die Schweiz regelte in der Verfassung bis 1983 außerdem noch die Kostenverteilung bei Einbürgerungen zwischen Bund und Kantonen. Heute heißt es, daß die StA Bundessache ist; die Kantone bürgern aber ein, nachdem der Bund zustimmte. Der Bund kann Mindestvorschriften für die Einbürgerung erlassen.

Die Bundesstaaten mit der genannten Zuständigkeitsklausel sind:

Afrika: Komoren, Tanzania

Asien: $\quad$ Indien, Malaysia, Pakistan, Ver. Arab. Emirate

Amerika: Argentinien, Brasilien, Kanada, Mexico, Vereinigte Staaten, Venezuela 
Australien: Australien

Europa: $\quad$ Bundesrepublik, Jugoslawien, Osterreich, Schweiz, SU, Tschechoslowakei

Den Bundesstaaten entsprechende Klauseln gibt es in Spanien dafür, daß die Regionen, bei Frankreich bzw. den Niederlanden dafür, daß Neukaledonien und Polynesien bzw. die Nied. Antillen f. d. StA nicht zuständig sind. Ferner ist in den Verfassungen v. Cook u. Niue vorgesehen, daß das alte StA v. Neuseeland weiter galt, das neue aber nur mit Zustimmung der Inseln auf diese erstreckt werden konnte.

\section{3) Einbürgerungsbehörde}

6 Staaten regeln in der Verfassung, welche staatliche Institution die Einbürgerung vornimme, nämlich entweder

der Präsident: Columbien, Finnland; oder

das Parlament: Norwegen, Mongolei, Belgien.

Der Kronrat ist in Monaco bei Einbürgerung u. Wiedererwerb zu hören.

\section{4) Diskriminierung}

Panama schloß Eingebürgerte von bestimmten Handelszweigen aus. Sierra Leone schloß Eingebürgerte von Parlamentssitzen aus und ließ Registrierte erst nach 25 Jahren zu, wobei solche Diskriminierung in einer anderen Verfassungsbestimmung ausdrücklich für zulässig erklärt wurde.

Norwegen dagegen ließ für Staatsämter ausdrücklich auch Eingebürgerte zu, falls sie die Landessprache sprechen.

Sri Lanka verbietet Unterscheidungen nach Erwerbsart (Geburt o. Registrierung): das gilt insb. für Tamilen.

Vier Staaten betonen, daß alle Staatsangehörigen auch Staatsbürger sind (China, Taiwan, Vietnam, Liechtenstein), d. h. daß jede Diskriminierung verboten ist, wie sie z. B. im Dritten Reich das Reichsbürgergesetz einführte.

\section{5) Einheit der StA}

Einige Staaten verkünden, aus je verschiedenen Gründen, daß die StA eine einheitliche sei:

Somalia tat dies, weil Brit. Somaliland sich 1960 für unabhängig erklärt und ein eigenes StAG erlassen hatte, das erst durch das somalische StAG v. 1962 aufgehoben worden war.

Zaire bestimmt, seine StA sei "une et exclusive". Die Einheit dürfte sich auf den Separatismus Katangas beziehen, das 1961 ein eigenes StA erlassen hatte. Die Exklusivität wird dadurch erläutert, daß Doppelstaatigkeit verboten ist (s. u.). Die gleiche Vorschrift wird im StAG v. 1981 in Art. 1 II wiederholt. 
Süd-Yemen hat in Art. 1 ein Vereinigungsgebot und sagt in Art. 2, daß Yemen ein Volk sei und daß die StA eine sei. Das dürfte bedeuten, daß Süd-Yemen allen StA'en NordYemens seine eigene StA zuspielt, ähnlich der BRD in Bezug auf die DDR oder wie früher Art. 1 IV der DDR-Verfassung v. 1949 ("Es gibt nur eine deutsche StA").

Die Sowjetunion meint mit einheitliche StA die Bundeszugehörigkeit, indem sie hinzufügt, daß jeder Bürger einer Unionsrepublik ein StA'er der SU sei. Eine solche Bestimmung war insb. deshalb nötig, weil die Ukraine und Weißrußland im Widerspruch zum Völkerrecht, selbständige Mitglieder der UNO sind. Ebenso bedeutet die "eine" StA der Ver. Arab. Emirate die Bundeszugehörigkeit.

\section{6) Diverses}

Australien hat die bloß noch historische Vorschrift, daß eine lokale Einbürgerung in eine brit. Kolonie als Einbürgerung für ganz Australien anerkannt wird.

Vier Staaten des Commonwealth (Dominica, St. Christopher, St. Lucia, St. Vincent) erwähnen in den Mantel-Orders zu ihren Verfassungen, daß ihre StA'en auch Commonwealth Citizens sind, welche Vorschrift Malaysia in seine Verfassung selbst aufnahm. Tanzania bestimmt in seiner Verfassung, daß das StAG v. 1962 nur mit 2/3 Mehrheit geändert werden darf, womit das StAG praktisch zu Verfassungsrang erhoben wird. Portugal führt als Grund der StA auch Verträge an; Spanien ermächtigt zu Doppelstaater-Abkommen mit ibero-amerikanischen Staaten und solchen, mit denen besondere Bindungen bestehen.

\section{Materielles Recht}

\section{A) Kernregelung}

Der Kern des StA-Rechts, die Erwerbs- und Verlustgründe, werden in den meisten Staaten des Commonwealth (26) und 6 weiteren in der Verfassung geregelt, wobei daneben für Sonderfragen (Einbürgerung, Aberkennung, Wiedererwerb, Verfahren) noch ein eigenes StAG besteht. Diese Regelung gilt nach Kontinenten wie folgt:

Afrika:

Botswana (aber StAB 1982 aufgehoben), Gambia, Ghana, Kenia, Mauritius, Nigeria, Seychellen, Uganda, Zambia, Zimbabwe. Dem Vorbild angeschlossen haben sich die 4 Bantu-Staaten (Bophutatswana, Ciskei, Transkei, Venda) sowie das anglophone Liberia.

Asien: Indien, Malaysia, Malediven, Singapur, Angeschlossen hat sich das früher unter brit. Oberhoheit stehende Nepal. 
Amerika: Antigua u. Barbuda, Bahamas, Barbados, Belize, Domiria, Grenada, Guyana, Jamaica, St. Christopher u. Nevis, St. Lucia, St. Vincent, Trinidad u. Tobago.

Australien: $\quad$ Fiji, Kiribati, Nauru, Papua Neuguinea, Solomonen, Tuvalu, Vanuatu

Europa: Malta, Zypern.

Das gleiche Prinzip gilt in den 17 ibero-amerikanischen Staaten, nämlich: Bolivien, Brasilien, Chile, Columbien, Costa Rica, Cuba, Domin. Republik, Ecuador, Guatemala, Honduras, Mexico, Panama, Paraguay, Peru, Salvador, Uruguay, Venezuela; sowie im frankophonen Haiti. Dem angeschlossen haben sich die früher spanischen Kolonien Äquatorialguinea und die Philippinen.

In 32 Staaten der Welt ist also das materielle StAR in der Verfassung geregelt. Ob das angesichts häufiger Staatsstreiche, die die Verfassung außer Kraft setzen, noch überall sinnvoll ist, mag bezweifelt werden. Der Grundgedanke war jedenfalls, die StA als eines der 3 Elemente des Staates in der Verfassung hervorzuheben und ihre Änderung zu erschweren.

\section{B) Einzelne Verlustgründe}

Weitaus am verbreitetsten ist hier ein Ausbürgerungsverbot:

1) Oft ist allerdings nur gesagt, daß eine Entziehung der StA nur im Rahmen des Gesetzes erlaubt ist (Kuwait, Qatar, Nord- u. Südyemen) bzw. nur in den vom Gesetz definierten Sonderfällen (Ver. Arab. Emirate). Diese Klauseln sind von geringem Wert, da durch Gesetz alle Ausbürgerungstatbestände eingeführt werden können, ja sogar Gesetze zur Ausbürgerung bestimmter Personen möglich sind. Verboten ist lediglich eine individuelle Ausbürgerung ohne Gesetzesgrundlage.

Bahrain sagt, daß Eingebürgerten die StA im Rahmen des Gesetzes stets entziehbar sind, Gebürtigen dagegen nur in zwei Sonderfällen (s. u.).

Sri Lanka führt die Sections des StAG auf, auf Grund derer Entziehung möglich ist.

2) Einige Staaten erlauben eine Entziehung bei Treubruch (Illoyalität) in Taten (Türkei, Nord-Zypern) oder bei Dienst in fremder Wehrmacht (nur bei Gebürtigen u. wenn ungenehmigt $=$ Monaco) oder bei Verstoß gegen Interessen, was durch Gesetz näher zu regeln ist (Griechenland).

Bahrain läßt eine Entziehung bei Gebürtigen nur bei Hochverrat zu, während sie bei Eingebürgerten unbeschränkt zulässig ist (s. o.). Jugoslawien hat eine sehr komplizierte Regelung: Bei Inlandswohnsitz ist eine Entziehung u. Ausweisung verboten. Bei Auslandswohnsitz ist sie nur nur auf Grund eines Bundesgesetzes und bei Besitz einer anderen StA möglich, wenn internationale oder andere Interessen Jugoslawiens geschädigt o. Bürgerpflichten nicht erfüllt sind.

Italien dagegen verbietet gerade jede Entziehung aus politischen Gründen - eingedenk des Faschismus. 
Drei der o. g. Staaten kennen neben jenen Gründen noch Besitz (Bahrain) bzw. freiwilligen Erwerb einer fremden StA (Griechenland, Monaco), letzteres hat die beiden Entziehungsgründe nur bei Gebürtigen, während bei Eingebürgerten das Gesetz unbeschränkt weitere Tatbestände aufstellen kann.

Zaire verbietet Doppelstaatigkeit: das könnte dahin ausgelegt werden, daß bei Erwerb einer fremden StA automatisch strafweise die bisherige StA erlischt.

3) Vier Staaten verbieten generell jede Ausbürgerung (Bundesrepublik, Tunis, Nicaragua; Spanien bei Gebürtigen).

Nicaragua verbietet es auch, das Recht auf Wechsel der StA zu bestreiten.

Die BRD sagt außerdem, daß ein Verlust der StA in übrigen Fällen nur erlaubt ist, wenn man dadurch nicht staatenlos wird.

Iran erlaubt Ausbürgerung und anderen Verlust der StA nur, wenn man eine andere StA besitzt.

4) Die Türkei sagt, daß bei Entziehung der StA, die also unbeschränkt zulässig ist, der Rechtsweg nicht ausgeschlossen werden darf.

\section{C) Einzelne Erwerbsgründe}

\section{1) Einbürgerung}

Dänemark u. Island bestimmen formell, daß jede Einbürgerung nur durch Einzelgesetze erfolgen darf. Luxemburg sagt, das Gesetz bestimme die Folgen der Einbürgerung. Finnland sagt, das Gesetz müsse Bedingungen und Verfahren der Einbürgerung festlegen. In Belgien verleiht nur die sog. große Einbürgerung politische Gleichstellung. Syrien sieht vor, daß ein Gesetz ehem. Syrer und Söhne arab. Länder erleichtert einbürgern soll. Die Bundesrepublik bürgert die sog. Statusdeutschen als Staatsangehörige 2. Klasse ein. Materiellen Gehalt hat die Vorschrift, daß Einbürgerung erst nach 5 Jahren Wohnsitz u. Eid erfolgen darf (Tonga) bzw. nach 2 Jahren (Argentinien), wobei hier Abkürzung möglich ist.

Einem Einbürgerungsanspruch gleich steht die Gewährung eines Optionsrechts der Briten auf Cocos und Christmas für die australische StA. Einer Zwangseinbürgerung gleich steht der automatische StA-Erwerb auf Wallis und Futuna, deren Bewohner bisher franz. Schutzgenossen gewesen waren, sowie auf Guam und den Nordmarianen, deren Bewohner US-Bürger wurden.

\section{2) Ius sanguinis oder ius soli}

Die Verfassung der USA (ebenso Guam, Nordmarianen und im Treuhandgebiet) enthält ein Bekenntnis zum ius soli (was detailliert in vielen Verfassungen des Commonwealth $u$. Lateinamerikas vorkommt, s. o.). Wer in Brunei von 7 Rassen geboren war, war StA'er (so bis 1963). Fünf Staaten bekennen sich zum ius sanguinis, und zwar bei Abstammung von beiden Eltern (Finnland, Burma, Türkei) bzw. nur beim Vater (Monaco, Brunei). 


\section{3) Gleichberechtigung der Frau im StAR}

In Island darf niemand wegen seines Geschlechts vom StA-Erwerb ausgeschlossen werden. In der Schweiz erwarben bis 1983 die Ehefrau und das Kind einer gebürtigen Schweizerin, wenn beide bei der Geburt Domizil in der Schweiz haben, die StA. Griechenland hat eine Gleichberechtigungsvorschrift, die in Art. 4 Abs. 2 unmittelbar vor einem StAB in Abs. 3 steht: diese generelle Vorschrift hat natürlich auch Auswirkungen auf das StAR, ebenso wie Art. 3 II GG.

\section{4) Erwerb durch Beruf}

In Osterreich wird durch Antritt einer Stellung als Hochschullehrer automatisch die StA erworben, in Griechenland durch Eintritt in die Klöster des Athos.

\section{5) Wiedereinbürgerung}

In der Bundesrepublik gewährt Art. 116 II GG einen Wiedereinbürgerungsanspruch für Deutsche, die unter dem Nationalsozialismus 1933-1945 ausgebürgert wurden. Dies ist eine Wiedergutmachungsvorschrift, insb. für Juden. Im übrigen hat nur Griechenland eine ähnliche Regelung bei StA-Entziehungen unter der Diktatur 1967-1975, nur daß hier eine Einzelprüfung vorgesehen ist und kein absoluter Anspruch.

\section{6) Indigenat}

Bei abhängigen Gebieten gibt es verschiedene Formen einer Gebietszugehörigkeit. So regelt Bermuda den dortigen Status brit. StA'er; Gibraltar verweist dagegen für den Gibraltarian Status auf eine Sonder-VO. Anguilla und die brit. Virgin Islands regeln die Zugehörigkeit ("belonging to"). Finnland regelt $\mathrm{f}$. d. Aaland-Inseln ausführlich ein echtes Indigenat, Dänemark für die Faröer nur ansatzweise. Cook und Niue regeln, wer ständiger Einwohner ist. Die 3 assoziierten Staaten der USA in der Südsee (Marschallinseln, Palau, Mikronesien) regeln eine eigene StA (Schutzangehörigkeit).

\section{Af rika}

\section{1) Ägypten}

11. 9.1971 (=): Verfassung der Arabischen Republik Ägypten

StA: Art. 6 (Verweisung auf Gesetz)

2) Äquatorialguinea

3. 8. 1982: Ley Fundamental de Guinea Ecuatorial

StA: Art. 9-13 (materiell) 


\section{3) Äthiopien}

ohne Verfassung

4) Algerien

22. 11. 1976: Ordonnance Nr. 97: Verfassung der Demokratischen Volksrepublik Algerien

StA: -

5) Angola

10. 11.1978 (11. 11.): Lei Constitucional da Republica Popular de Angola StA: -

6) Benin

26. 8. 1977: Loi Fondamentale de la République Populaire du Benin StA: -

\section{7) Bophutatswana}

6. 12. 1977 (=): Republic of Bophutatswana Constitution Act, Nr. 18 StA: Sec. 80 (Originärer Erwerb; i. ü. Verweisung auf Gesetze)

\section{8) Botswana}

20. 9.1966 (30. 9.): (UK) Botswana Independence Order, S. I. Nr. 1171

StA: Sec. 20-30 (materiell)

Änderungen der StA:

1) 29. 1. 1969, Citizenship (Specific Date) Act, Nr. 2, ändert Sec. 23

2) 18. 8. 1969, Constitution (Amd. and Suppl. Prov.) Act Nr. 30. ändert Sec. 25, 26; hebt auf Sec. 23

3) 2. 7. 1970, Constitution Amd. Act, Nr. 25, ändert Sec. 25, 26

4) 25. 9. 1973, Revision of the Laws Act, Nr. 15, läßt Nummer für aufgehobene Sec. 23 aufrücken

5) 23. 9. 1982, Const. Amd. Act Nr. 32, hebt auf Sec. 20-29 n. F., nachdem StAG Nr. 25/1982 ergangen

9) Burkina-Faso

ohne Verfassung

10) Burundi

20. 11. 1981: Decrète-Loi Nr. 1-23: Constitution

StA: Art. 8 (Verweisung auf Gesetze)

11) Ciskei

4. 12. 1981 (=): Republic of Ciskei Constitution Act, Nr. 20

StA: Sec. 67 (materiell)

12) Djibouti

27. 6. 1977 (=): Lois Constituionelles Nr. 1 u. 2

StA: -

13) Elfenbeinküste

31. 10. 1960 (3. 11.): Loi 356: Constitution de la République StA: - 


\section{4) Gabun}

15. 4. 1975: Constitution de la République

StA: -

15) Gambia

24. 4. 1970 (=): Constitution of the Republic of Gambia Act, Nr. 1

StA: Sec. 3-12 (materiell)

16) Ghana

31. 12. 1982: Prov. Nat. Def. Council (Establ.) Procl. (Suppl. and Conseq. Prov.)

Law Nr. 42

StA: Sec. 51 (materiell) u. 60 (formell)

17) Guinea

10. 10. 1958 (12. 11.): Loi 4: Constitution de la République de Guinée

StA: -

18) Guinea-Bissau

16. 5. 1984: Constituição da República da Guiné-Bissan

StA: -

19) Kamerun

2. 6. 1972 (=): Decret Nr. 270: Constitution de la République du Cameroun StA: -

20) Kapverde

7. 10. 1980 (13. 10.): Lei 3: Constituição de las islas de Capo Verde

StA: Art. 29 (aufgehoben durch Lei 2 v. 12. 2. 1981)

21) Kenia

10. 4. 1969 (18. 4.): Constitution of Kenya Act, Nr. 5

StA: Sec. 87-98 (materiell)

22) Komoren

1. 10. 1978: Constitution de la République Fédérale Islamique des Comores StA: Art. 30 Z. 9 (Bundeszuständigkeit)

23) Kongo

8. 7. 1979: Constitution de la République Populaire du Congo

StA: -

24) Lesotho

13. 4. 1973: Lesotho Order, Nr. 13

StA: -

25) Liberia

19. 10. 1983: Constitution of the Republic of Liberia

StA: Sec. 27-28 (Grundzüge)

26) Libyen

11. 12. 1969: Verfassungsproklamation

StA: - 
27) Madagaskar

30. 12. 1975: Constitution de la République Démocratique de Madagascar StA: -

28) Malawi

14. 6. 1966 (6. 7.): Republic of Malawi (Constitution) Act, Nr. 2

StA: Sec. 7 der Constitution (UB u. Ermächtigung)

29) Mali

25. 4. 1974 (1. 7.): Constitution de la République du Mali

StA: -

30) Marokko

10. 3. 1972: Verfassung des Königreichs Marokko

StA: -

31) Mauritius

4. 3.1968 (12. 3.): (UK) Mauritius Independence Order in Concil S. I. ohne Nr.

StA: Sec. 20-27 der Constitution (materiell)

32) Mauretanien

4. 1. 1980: Charte constitutionelle du Comité Militaire de Salut National de Mauritanie

StA: -

33) Mozambique

20. 6. 1975 (25. 6): Constituição de la Republica Popular de Mocambique StA: -

34) Niger

8. 11. 1960: Constitution de la République du Niger (1974 suspendiert, nicht aufgehoben)

StA: -

35) Nigeria

21. 9. 1978 (1. 10. 79): Constitution of the Federal Republic of Nigeria (Enactment) Decree, Nr. 25

StA: Sec. 23-29 (materiell), Sec. 268 (frühere StA)

36) Rwanda

20. 12. 1978: Constitution de la République' Rwanda

StA: Art. 5 (Verweisung auf Gesetze)

37) Saõ Tomé e Principé

5. 1.1978 (=): Constituicão Politica de Saõ Tomé e Principé

StA: -

38) Senegal

7. 3. 1963: Loi 32: Constitution de la République

StA: Art 56 (Parlament zuständig)

39) Seychellen

23. 3. 1979 (5. 6.): Constitution of the Republic of Seychelles Decree, Nr. 12

StA: Sec. 9-16 der Constitution (materiell) 
40) Sierra Leone

14. 6. 1978 (=): Constitution of Sierra Leone Act, Nr. 12

StA: Sec 17 (4, h) und 44 (a, und Proviso): Nur StA-Diskriminierungen

41) Somalia

August 1979: Verfassung der Demokratischen Republik Somalia

StA: Art. 4 I (Einheit der StA), II (Verweisung auf Gesetze)

42) Südafrika

22. 9. 1983 (3. 9. 84): Republic of South Africa Constitution Act, Nr. 110 StA: -

43) Sudan

14. 4. 1973 (8. 5.): Permanent Constitution of the Democratic Republic of the Sudan

44) Swaziland

12. 4. 1973 (=): King's Proclamation

StA: -

45) Tanzania

25. 4. 1977 (26. 4.): Constitution of Tanzania

StA: Schedule 1 (StAG v. 1962 nur mit 2/3 Mehrheit änderbar)

Schedule 2, § 6 (Union matters)

46) Togo

30. 12. 1979 (13. 1. 80): Constitution de la République

StA: -

47) Transkei

1976 (26. 10.): Republic of Transkei Constitution Act, Nr. 15

StA: Sec. 57-59 (materiell)

48) Tschad

29. 9.1982 (18. 10.): Acte fondamental de la République, Dekret Nr. 25

StA: -

49) Tunesien

1. 6. 1959 (=): Constitution de la République Tunisienne

StA: Art. 11 (Verbot der Ausbürgerung = Expatrie); Art. 34 Z. 3 (Gesetzesvorbehalt) erst ab 1976!

Änd.: 8. 4. 1976, Loi Nr. 37 ändert Art. 34 völlig, indem Gesetzesvorbehalt u. a. für StA eingeführt.

50) Uganda

8. 9.1967 (=): Constitution of the Republic of Uganda

StA: Sec. 4-7 (materiell)

51) Venda

30. 11. 1979: Republic of Venda Constitution Act, Nr. 9

StA: Sec. 59-60 (materiell) 
52) Zaire

15. 2. 1978 (1. 3.): Lei 10: Constitution de la République de Zaire

StA: Art. 11 I (Einheit der StA: Verbot der Soppelstaatigkeit)

Art. 11 II (Verweisung auf Gesetze)

53) Zambia

25. 8. 1973 (=): Constitution of Zambia Act, Nr. 27

StA: Sec. 5-12 de Constitution (materiell); Sec. 3 des Mantelgesetzes (widerruft Indep. Act und Order v. 1964, die StAB'en hatten)

54) Zentralafrika

5. 2. 1981 (=): Constitution de la République Centrafricaine

StA: -

55) Zimbabwe

6. 12. 1979 (18. 4. 80): (UK) Zimbabwe Constitution Order, S. I. Nr. 1600

StA: Sec. 4-10 (materiell)

Änd.: 1981, Act No. 3, hebt auf Sec. 8, ändert Sec. 9

2. Asien

1) Afghanistan

21. 4. 1980: Grundprinzipien der Demokratischen Verfassung Afghanistans

StA: Art. 27 (Verweisung auf Gesetz)

2) Bahrain

26. 5. 1973: Verfassung des Staates Bahrain

StA: Art. 17 I (Verweisung auf Gesetz; Ausbürgerung Gebürtiger nur bei Hochverrat u. Doppelstaatlichkeit)

Art. 17 II (Eingebürgerten darf StA nur im Rahmen des Gesetzes entzogen werden)

3) Bangladesh

4. 11. 1972 (16. 12.): Verfassung der Volksrepublik Bangladesh (suspendiert 1982)

StA: Art. 6 (Verweisung auf Gesetz)

4) Bhutan

27. 6. 1969 Verfassungskonvention

StA: -

5) Brunei

29. 9. 1959 Constitution of the State of Brunei, S. 97

StA: Art. 2 Ziff. 4 u. 5 (Definition: Brit. Subject; Brunei'er)

Art. 29 II (ursprüngl. StA)

Änd. v. Art. 29 II durch Enactment Nr. 2 v. 13. 5.1963 ("Untertan des

Sultans" in Art. 29 I ist, wer nach StAG Staatsangehöriger) 


\section{6) Burma}

3. 1. 1974: Verfassung der Sozialistischen Republik der Union von Burma

StA: Art. 145 (ursprüngl. StA)

Art. 146 (Verweisung auf Gesetz)

\section{7) China}

4. 12. 1982: Verfassung der Volksrepublik China

StA: Art. 33 I (alle Staatsangehörigen sind Staatsbürger)

8) China/Taiwan

1. 1. 1947 (25. 12.): Verfassung der Republik China

StA: Art. 3 (Staatsangehörige sind Staatsbürger)

9) Indien

26. 11. 1949 (26. 1. 50, aber Sec. 5-9 am 26. 11. 49) Constitution of India

StA: Art. 5-11 (materiell)

Änd.: Art. 7 Proviso II geändert f. Kaschmir durch indische Order Nr. 48 v. 14. 4. 1954

10) Indonesien

17. 8. 1945 (=): Verfassung der indonesischen Republik

StA: Art. 26 I (StA'e = Gebürtige u. Eingebürgerte)

Art. 26 II (StA wird durch Gesetz geregelt)

Änd.: Die Verfassung war 17. 8. 1950-5. 7. 1959 außer Kraft

11) Irak

16. 7. 1970: Vorläufige Verfassung der Republik Irak

StA: Art. 6 (StA wird durch Gesetz geregelt)

12) Iran

15. 11. 1979: Verfassung der islamischen Republik Iran

StA: Art. 41-42 (Verschiedenes)

13) Israel

Keine Verfassung, nur einzelne Grundgesetze (ohne StAB)

14) Japan

3. 11. 1946 (3. 5. 47): Verfassung v. Japan

StA: -

15) Jordanien

1. 1.1952 (8. 1.): Verfassung des Haschimitischen Königreichs Jordanien

StA: Art. 5 (StA wird durch Gesetz geregelt)

16) Kambodscha

27. 6. 1981: Verfassung der Volksrepublik Kampuchea

StA: -

17) Korea (Nord)

27. 12. 1972: Sozialistische Verfassung der Demokratischen Volksrepublik Korea StA: - 
18) Korea (Süd)

26. 11. 1972: Verfassung der Republik Korea

StA: Art. 2 I (StA wird durch Gesetz geregelt)

19) Kuwait

11. 11. 1962 (Jan. 1963): Verfassung des Staates Kuwait

StA: Art. 27 (StA wird durch Gesetz geregelt; Entzug nur im Rahmen des StAG)

20) Laos

keine Verfassung

21) Libanon

23. 5. 1926: Verfassung der Libanesischen Republik

StA: Art. 6 (StA wird durch Gesetz geregelt)

22) Malaysia

5. 8. 1957 (30. 8.): Federal Constitution: Constitution of Malaya

StA: Art. 14-31 (materiell), Art. 155 (Commonwealth), Art. 160 II (Definition), Art. 170 (UB), Schedule 1 (Eid), Schedule 2 (StA i. a.), Schedule 9, Liste I, Nr. 5 (Bundeszuständigkeit)

Änderungen:

1) 26. 5. 1960, CAA Nr. 10, ändert Art. 16 u. 23

2) 20. 2. 1962, CAA Nr. 20, ändert Art. 14, 15, 18, 19, 21, 23-26, 155, 160 II, Schedule 1; fügt ein Art. 15 A, 26 A, 26 B; hebt auf Art. 17 u. 20

3) 26. 8. 1963, CAA Nr. 25, ändert Art. 16 u. 18, hebt auf Art. 170

4) 16. 9. 1963, Malaysia Act Nr. 26, ändert Art. 14-16, 18, 19, 22, 24, 25, 26 A-B, 28, 30, 31; fügt ein Art. 16 A, 19 A, 28 B, 31 A, 31 B, hebt auf Art. 21, ändert Schedule 2

5) 29. 7. 1964, CAA Nr. 19, hebt auf Art. 26 (4)

6) 22. 6. 1965, Constitution and Malaysia Act (Amd) Act, Nr. 31, ändert Art. $160 \mathrm{II}$

7) 9. 8. 1965, Constitution and Malaysia (Singapur Amd) Act, Nr. 53 widerruft alle Vorschriften betr. Singapur, da nach Sec. 12 für Singapur die StA Malaysias endet

8) 19. 9. 1966, CAA Nr. 59, ändert Art. 14-16, 16 A, 18, 19, 26 A, 28 A, 30, 160 II, Schedule 2; hebt auf Art. 19 A, 30 A, 30 B

9) 25. 2. 1976, CAA Nr. A 354, ändert Art. 24-25 und ersetzt überall "Borneo States" durch States of Sabah and Sarawak"

23) Malediven

11. 11. 1968: Verfassung der Republik

StA: Art. 2-4 (materiell)

Änderung: 2. 2. 1972, Amendment, ändert Art. 4 (5)

24) Mongolei

6. 7. 1960: Verfassung der Mongolischen Volksrepublik

StA: Art. 34 (t) (Präsidium verleiht und hebt auf StA) 
25) Nepal

16. 12. 1962: Verfassung des Königreichs Nepal

StA: Sec. 7-8 (materiell)

Änderungen:

1) 12. 12. 1975, zweite Änderung, ändert Sec. 8 (2, d)

26) Oman

2) 15. 12. 1980, dritte Änderung, ändert Sec. 8 (2, d); fügt ein Sec. 8 (2, e)

\section{keine Verfassung}

27) Pakistan

12. 4. 1973 (14. 8.): Verfassung der islamischen Republik Pakistan

StA: Schedule 4 I Nr. 4 (Federal List: Nationality, Citizenship, Naturalisation),

28) Philippinen

17. 1.1973 (=): Constitution of the Republic of the Philippines

StA: Art. III (Sec. 1-4) (materiell)

29) Qatar

2. 4. 1970: Provisional Constitution for Qatar

StA: Art. 4 I (StA soll durch Gesetz geregelt)

30) Saudiarabien

Art. 4 II (Aberkennung der StA nur in Fällen des Gesetzes)

keine Verfassung

31) Singapur

29. 8. 1963 (16. 9.): (UK) Constitution of the State of Singapur, S. I. Nr. 1493

StA: Art 53-69; Schedule 2 (Eid); Schedule 3 (StA-Verfahren)

Änderungen:

1) 7. 3. 1966, Modification of Laws (Constitution of Singapore) Order (MLCS) Nr. S 50, hebt auf Art. 53 (3), 55 (2), 56, 61 (5), 67

2) 9. 12. 1966, MLSC Order Nr. S 259, fügt wieder eine Clause 2 zu Art. 55 hinzu; fügt 60 (3) an, fügt 61 (3 A) u. 63 A ein, ändert Schedule 2

3) 10. 3. 1967, MLSC Order S 58, ändert Art. 54

4) 3. 5. 1967, MLSC Order S 88, ändert Art. 57 (1), 60, 63 A (3), Schedule 2; ändert in Schedule 3 Sec. 7 u. hebt auf Sec. 16

5) 25. 7. 1968, MLSC Order S 214, ändert Art. 57

6) 6. 8. 1968, CAA Nr. 21, ändert Art. 55, 60 (3), 63 (2), 66

7) 29. 12. 1969, CAA Nr. 19, ändert Art. 63 (2)

8) I.K. 4. 5. 1979, CAA Nr. 10, fügt ein Art. $60 \mathrm{~A}$

9) 31. 3. 1980, Official reprint, verändert Verfassung total unter Einarbeitung der weitergeltenden Vorschriften der Verfassung Malaysias. Die StA sind jetzt Part X, Art. 120-141; Schedule 2-3 bleiben so.

32) Sri Lanka

31. 8. 1978 (7. 9.): Constitution of the Democratic Socialist Republic of Sri Lanka StA: Art. 26 (Verschiedenes) 


\section{3) Syrien}

31. 1. 1973 (13. 3.): Verfassung der Syrischen Arabischen Republik

StA: Art. 43 (StA durch Gesetz geregelt; Besonderes)

34) Thailand

22. 12. 1978 (=): Verfassung des Königreichs Thailand

StA: -

35) Vereinigte Arabische Emirate

18. 7. 1971: Vorläufige Verfassung der Vereinigten Arabischen Emirate

StA: Art. 8 (Diverses), Art. 120 Nr. 16 (Bundeszuständigkeit)

36) Vietnam

18. 12. 1980 Verfassung der Sozialistischen Republik Vietnam

StA: Art. 53 (Staatsangehörige sind Staatsbürger)

37) Yemen (Nord)

28. 12. 1970 (30. 12.): Verfassung der Arabischen Republik Yemen (suspendiert)

StA: Art. 20 (StA wird durch Gesetz geregelt; Ausbürgerungsverbot außer durch Gesetz)

38) Yemen (Sïd)

30. 11. 1970: Verfassung der Demokratischen Volksrepublik Yemen

StA: Art. 2 (nur eine StA), Art. 43 (Ausbürgerungsverbot außer durch Gesetz)

\section{Australien und Ozeanien}

1) Australien

9. 7. 1900 (1. 1. 01): (UK) Commonwealth of Australia Constitution Act, 63/4 Vict.,.c. 12

StA: Sec. 34 (ii) der Constitution (auch bloße Kolonial-Einbürgerung anerkannt) Sec. 51 Ziff. 19 (Bundeszuständigkeit)

2) Fiji

30. 9.1970 (10. 10.): (UK) Fiji Independence Order in Concil, S. I. Nr. 1970

StA: Sec. 19-26 (materiell)

3) Kiribati (Gilbert Inseln)

26. 6.79 (12. 7.): (UK) Kiribati Independence Order, S. I. Nr. 719

StA: Sec. 19-29 (materiell)

4) Nauru

29. 1. 1968 (31. 1.): Constitution of Nauru

StA: Art. 71-76 (materiell)

Änd.: 17. 5. 1968, ändert Art. 74; fügt neue Schedule 5 hinzu (nach VI ist Art. 71 u.

72 (1) nur mit 2/3 Mehrheit änderbar)

5) Neuseeland

30. 6. 1852: (UK) New Zealand Constitution Act, 15/6 Vict. 72

StA: - 


\section{6) Papua Neuguinea}

15. 8. 1975 (16. 9.): Constitution of Papua New Guinea

StA: Sec. 64-81 (materiell) Schedule 2 (6) i. V. m. Schedule 5 (1) (Weitergeltung austral. StA-Rechts)

Änderung: 23. 3. 1977, Constitution Amendment Act, Nr. 5 ändert Sec. 76

7) Solomon Inseln

31. 5.1978 (7. 7.): (UK) Solomon Islands Independence Order, S. I. Nr. 783

StA: Sec. 20-26 (materiell)

8) Tonga

4. 11. 1875: Constitution of Tonga

StA: Sec. 29 Satz 1 (Einbürgerung)

Änderungen:

1) 21. 9. 1912, Amendment Act Nr. 35, ändert Sec. 29

2) 12. 6. 1979, Amendment Act, ändert Sec. 29

9) Tuvalu (Ellice Inseln)

25. 7. 1978 (1. 10.): (UK) Tuvalu Independence Order, S. I. ohne Nr.!

StA: Sec. 19-27 (materiell)

10) Vanuata (Neue Hebriden)

30. 7. $1980(=)$ : Constitution of the New Hebrides

StA: Art. 9-14 (materiell)

11) Westsamoa

28. 10. 1960 (1. 1. 62): Constitution of the Independent State of Western Samoa StA: -

\section{Amerika}

1) Antigua und Barbuda

31. 7. 1981 (31. 10.): (UK) Antigua und Barbuda Constitution Order S. I. Nr. 1106

StA: Sec. 111-118 der Constitution (materiell); $§ 10$ der 2. Schedule der Order (Commonwealth Citizen)

2) Argentinien

25. 9.1860 (1. 10.): Constitución de la Nación Argentina

StA: Art. 20-21 (Einbürgerung), Art. 67 Ziff. 11 (Bund zuständig), Art. 108 (Provinzen unzuständig)

3) Bahamas

20. 6. 1973 (10. 7.): Bahamas Independence Order, S. I. Nr. 1080

StA: Sec. 3-14 der Constitution (materiell)

4) Barbados

22. 11. 1966 (30. 11.): (UK) Barbados Independence Order, S. I. Nr. 1455

StA: Sec. 2-10 der Constitution (materiell)

Änd. der StA: 4. 10. 1974, Constitution Amendment Act Nr. 34, ändert Sec. 3, 6, 7 


\section{5) Belize}

20. 9. 1981 (21. 9.): Constitution of Belize Act, Nr. 14

StA: Sec. 23-29 (materiell)

6) Bolivien

2. 2. 1967 (3. 2.): Constitución Política del Estado de Bolivia

StA: Art. 36-39 (materiell)

7) Brasilien

17. 10. 1969 (30. 10.): Constituição do Brasil

StA: Art. 3 XVII (o) (Bundeszuständigkeit), Art. 145-146 (materiell)

8) Chile

8. 8. 1980 (11. 3. 81) Constitución de Chile

StA: Art. 10-12 (materiell)

9) Columbien

4. 8.1886 (6. 9.): Constitución política de Colombia

StA: Art. 8-9 (materiell), Art. 13 II (Wehrdienstbefreiung), Art. 120 Ziff. 17 (Präsident zuständig für Einbürgerung)

Änd. der StA: 1. 8. 1936, Acto Legislativo, ändert Art. 8-9

10) Costa Rica

7. 11. 1949 (8. 11.): Constitución de Costa Rica

StA: Art. 13-17 (materiell), UB 1 (zu Art. 13)

Änd. der StA: 9. (12.) 5. 1961, Decreto Legislativo 2739, ändert Art. 16

11) Cuba

24. 2. 1976 (=): Constitución de la República de Cuba

StA: Art. 28-33 (materiell)

12) Dominica

25. 7. 1978 (3. 11.): (UK) Commonwealth of Dominica Constitution Order, S. I.

Nr. 1027

StA: Sec. 97-102 der Constitution (materiell), § 13 der 2. Schedule der Order (Commonwealth Citizen)

13) Dominikanische Republik

28. 11. 1966: Constitución de la República Dominicana

StA: Art. 11 (materiell)

14) Ecuador

23. 5. 1977 (10. 8. 79): Constitución política de la República del Ecuador

StA: Art. 5-11 (materiell)

Änd. der StA: 24. 8. 1983, Emenda constitucional, ändert Art. 6, 7, 11

15) Grenada

19. 12. 1973 (7. 2. 74): (UK) Grenada Constitution Order, S.I. Nr. 2155

StA: Art. 94-100 (materiell)

16) Guatemala

27. 4. 1982 (28. 4.): Estatuto Fundamental del Gobierno

StA: Art. 9-17 (materiell) 


\section{7) Guyana}

20. 2. 1980 (6. 10.): Constitution of the co-operative Republic of Guyana, Act Nr. 2 StA: Art. 41-49 (materiell)

18) Haiti

27. 8. 1983 (=): Constitutión de la République d'Haiti

StA: Art. 9-12 (materiell), Art. 15-16 (Einbürgerung), 17 (Wiedererwerb), 18 (Doppelstaater)

19) Honduras

11. 1. 1982 (20. 1.): Constitución de la República de Honduras, Decreto Nr. 131

StA: Art. 22-29 (materiell)

20) Jamaica

23. 7. 1962 (6. 8.): (UK) Jamaica Constitution Order, S.I. Nr. 1550

StA: Sec. 3-12 der Constitution (materiell)

21) Kanada

29. 3. 1982 (=): (UK) Canada Act, c. 11 (Constitution als Schedule B, i.K. 17. 4. 82)

StA: Sec. 91 Ziff. 25 des British North America Act, 1867 (Bundeszuständigkeit); dies britische Gesetz gilt z. T. noch weiter

22) Mexico

31. 1. 1917 (1. 5.): Constitución de los Estados Unidos de Mexico

StA: Art. 30 (Erwerb), 37 (A) (Verlust), 73 Ziff. 16 (Bundeszusändigkeit)

Änd.: 1) 18. 1. 1934, ändert Art. 30 u. 37

2) 6. 12. 1969, ändert Art. 30 (A II)

3) 27. 12. 1974, ändert Art. 30 (B II)

23) Nicaragua

21. 8. 1979: Estatuto de Derechos y Guarantias del Pueblo de Nicaragua, Dekret Nr. 52

StA: Art. 26 (Jeder hat Recht auf StA; niemand darf StA o. Recht zu wechseln aberkannt werden)

24) Panama

12. 5.1983 (6. 6.): Constitución Política de la República de Panamá

StA: Art. 8-16 (materiell), 288 (Handel nur f. bestimmte StA'e)

25) Paraguay

25. 8. 1967 (=): Constitución de la República de Paraguay

StA: Art. 24-30 (materiell), 36-37 (Allgemeines)

26) Peru

12. 7. 1979 (28. 7. 80): Constitución política del Peru

StA: Art. 89-94 (materiell)

27) Salvador

15. 12. 1983 (20. 12.): Dekret Nr. 38: Constitución Política de la República de El

Salvador

StA: Art. 90-100 (materiell) 


\section{8) St. Christopher und Nevis}

22. 6.1983 (23. 6.): Saint Christopher and Nevis Constitution Order, S.I. Nr. 881 StA: Sec. 90-95 (materiell); § 11 der 2. Schedule zur Order (Commonwealth Citizens)

29) St. Lucia

20. 12.1978 (22. 2. 79): (UK) Saint Lucia Constitution Order, S.I. Nr. 1901

StA: Sec. 99-104 (materiell), § 11 der 2. Schedule zur Order (Commonwealth Citizen)

30) St. Vincent und Grenadinen

26. 7. 1979 (27. 10.): (UK) Saint Vincent Constitution Order, S.I. Nr. 916

StA: Sec. 90-95 (materiell); § 12 der Schedule zur Order (Commonwealth Citizen)

31) Surinam

24. 11. 1975 (25. 11.): Grondwet der Republik Suriname

StA: Art. 2 I (Verweisung auf Gesetze)

32) Trinidad und Tobago

29. 3. 1976 (31. 7.): Constitution of the Republic of Trinidad and Tobago Act, Nr. 4

StA: Sec. 15-21 (materiell)

33) Uruguay

24. 8. 1966 (15. 2. 67): Constitución de la República Oriental del Uruguay

StA: Art. 73-76 (Erwerb), 81 (Verlust)

34) Venezuela

23. 1. 1961 (=): Constitución de la República de Venezuela

StA: Art. 35-42 (materiell), Art. 136 Ziff. 4 (Bundeszuständigkeit) UB 3 (zu Art. 36 II), UB 4 (Widerruf, Verfahren)

35) Vereinigte Staaten

17. 9.1787 (1. 7. 89): Constitution of the United States of America

StA: Art. I, Sec. 8 (Einbürgerung ist Bundessache)

Änd.: 9. (28.) 7. 1868, XIV. Amendment (ius soli unbeschränkt)

\section{Europa}

1) Albanien

28. 12. 1976 (=): Gesetz Nr. 5506: Verfassung der Sozialistischen Volksrepublik Albanien

StA: Art. 38 (StA'er ist, wer StA gemäß Gesetz hat)

2) Andorra

keine Verfassung

3) Belgien

7. 2. 1831 (25. 2.): Constitution du Royaume de Belgique

StA: Art. 4-5 (Verschiedenes), Art. 133 (UB, Option) 
Änd.: Die Angabe bei Franz, Staatsverfassungen 1950, S. 52-53, Art. 5 II sei 1921 gestrichen, ist falsch. Alle obigen 3 Artikel wurden niemals geändert.

4) Bulgarien

8. 5. 1971 (18. 5.): Verfassung der Volksrepublik Bulgarien

StA: Art. 34 (StA wird durch Gesetz geregelt)

5) Dänemark

5. 6.1953 (=): Lov Nr. 169: Danmarks Riges Grundlov

StA: $§ 44$ I (Einbürgerung nur aufgrund des Gesetzes)

6) Deutschland (BRD)

23. 5. 1949 (24. 5.): Grundgesetz für die Bundesrepublik Deutschland

StA: Art. 16 I (StA-Verlust), Art. 73 Z. 2 u. 74 Z. 8 (Zuständigkeit), Art. 116 (Besonderes)

7) Deutschland (DDR)

6. 4. 1968 (9. 4.): Verfassung der Deutschen Demokratischen Republik

StA: Art. 19 IV (StA wird durch Gesetz geregelt)

8) Finnland

17. 7. 1919: Regierungsform

StA: § 4 I (Abstammung), II (Einbürgerung), § 31 (Präsident verleiht u. entläßt)

9) Frankreich

4. 10. 1958 (5. 10.): Constitution de la République Française

StA: Art. 34 II (Regelung durch Gesetz)

10) Griechenland

9. 6. 1975 (11. 6.): Verfassung von Griechenland

StA: Art. 4 III (Verlustgründe), Art. 105 I, 3 (Athos), Art. 111 Z. 5-6 (UB)

11) Großbritannien

keine Verfassung

12) Irland

1. 7. 1937 (29. 12.): Verfassung von Eire

StA: Art. 9 (Diverses)

13) Island

23. 5. 1944 (17. 6.): Gesetz Nr. 33: Verfassung der Republik Island

StA: Art. 68 Satz 1 (Einbürgerung nur aufgrund eines Gesetzes)

14) Italien

27. 12.1947 (1. 1. 48): Costituzione de la Repubblica Italiana

StA: Art. 22 (Ausbürgerungsverbot aus politischen Gründen)

15) Jugoslawien

21. 2. 1974 (=): Verfassung der Sozialistischen Bundesrepublik Jugoslawien

StA: Art. 200 II-III (Ausbürgerung), Art. 249 (Bundes- u. Länder-StA)

16) Liechtenstein

5. 10. 1921: Verfassung des Fürstentums Liechtenstein

StA: Art. 29 (Landesangehörige haben Bürgerrecht), Art. 30 (Gesetze bestimmen StA) 
17) Luxemburg

17. 10. 1868: Constitution du Grand-Duché de Luxembourg

StA: Art. 9 I (Verweist auf CC), Art. 10 (Einbürgerung)

Änderung: 6. 5. 1948, Verfassungsänderungsgesetz, ändert Art. 10

18) Malta

2. 9.1964 (21. 9.): (UK): Malta Independence Order, S. I. Nr. 1398

StA: Sec. 23-32 der Constitution

Änderungen:

1) 29. 10. 1965 Constitution of Malta (Application of Sec. 29) Act, Nr. 41, ändert Sec. 29

2) 25. 10. 1966, CAA, Nr. 37, ändert Sec. 28

3) 14. 3. 1967, Constitution of Malta (Application of Sec. 29) Act, Nr. 9, ändert Anwendung v. Sec. 29

4) 13. 12. 1974 CAA, Nr. 58, ändert Sec. 24, 27, 28, 32; gibt UB

5) 25. 3. 1977 , CAA, Nr. 10, ändert Sec. 32

19) Monaco

15. 12.1962 (=): Constitution de la Principauté de Monaco

StA: Art. 18 (Diverses), Art. 77 II (Kronrat bei Einbürgerung u. Wiedererwerb hören)

20) Niederlande

24. 8. 1815 (28. 8.): Grondwet voor het Koninkrijk der Nederlanden

StA: siehe Änderungen:

1) 14. 11. 1848, eines der 12 Änderungsgesetze führt in den neunumerierten Text erstmals eine StAB ein: Art. 7 I (Verweisung auf Gesetz), II (Einbürgerung)

2) 15. 11. 1887, eines der 11 Änderungsgesetze fügt zu Art. 7 einen Abs. III (Erstreckung von Einbürgerung) hinzu und numeriert ihn um in Art. 6

3) 9. 12. 1922, eines der 10 Änderungsgesetze faßte Art. 6 I-II neu

4) 19. 1. 1983, eines der 35 Änderungsgesetze (StBl Nr. 20), die am 17.2. 83 in Kraft traten, hob in Art. III den alten Art. 6 auf und führte einen neuen Art. 2 ein, dessen Ziff. 1 lautete: "Das Gesetz bestimmt, wer Niederländer ist".

21) Norwegen

4. 11. 1814: Grundlov

StA: $§ 75$ lit. 1 (Storting bürgert ein), § 92 (Indigenatsvoraussetzungen für Ämter) Änderungen v. § 92 durch Gesetze 17) 15.6. 1878

2) 4. 6. 1892

3) 19. 3. 1901

4) 15.5 .1919

5) 28.6 .1952

22) Osterreich

1. 10. 1920 (10. 11.): Bundesverfassung

StA: Art. 6 I-IV (Landesbürgerschaft; Erwerb durch Hochschullehrer), Art. 11 Ziff. 1 (Bundeszuständigkeit) 
Änderung: Art. 6 I-III sind seit 1945 unanwendbar, da es seitdem keine Landesbürgerschaft mehr gibt. $\S 1$ des StBG v. 1965 behält Landesbürgerschaft einem Bundesverfassungsgesetz vor, das bisher nicht ergangen ist.

23) Polen

22. 7. 1952 (=): Verfassung der Polnischen Volksrepublik

StA: -

24) Portugal

2. 4. 1976 (25. 4.): Constituição da República Portuguesa

StA: Art. 4 (StA'e sind die durch Gesetz oder internat. Konvention)

25) Rumänien

21. 8. 1965: Verfassung der Sozialistischen Republik Rumänien

StA: -

26) San Marino

keine Verfassung

27) Schweden

28. 2. 1974 (1. 1. 75): Regeringsform

StA: Kap. 8, § 2 Ziff. 1 (StA wird durch Gesetz geregelt)

28) Schweiz

29. 5. 1874: Bundesverfassung der Schweizerischen Eidgenossenschaft

StA: Art. 43-45 (Diverses), Art. 54 IV (durch Ehe erwirbt Frau Heimatrecht des

Mannes), Art. 68 (Heimatlose), Art. 110 II (Bundesgericht zuständig)

Änderungen:

1) 25. 5. 1928, Verfassungsänderungsgesetz betr. Art. 44, ändert I-II, fügt hinzu III-VI

2) 24. 6. 1983, Verfassungsänderungsgesetz, faßt Art. 44 völlig neu (nur noch 3 Absätze), hebt auf Art. 54 IV

29) Sowjetunion

7. 10. 1977 (9. 11.): Verfassung der UdSSR

StA: Art. 33 (Bundeszuständigkeit; Regelung durch Gesetz; Schutz im Ausland)

30) Spanien

27. 12.1978 (29. 12.): Constitución de España

StA: Art. 11 (Diverses), Art. 149 Ziff. 2 (Staat, nicht Regionen zuständig)

31) Tschechoslowakei

27. 10. 1968 (1. 1. 69): Verfassungsgesetz Nr. 143 über die Föderation

StA: Art. 5 (Diverses)

32) Türkei

18. 10. 1982 (9. 11.): Gesetz Nr. 2709: Verfassung der Türkischen Republik

StA: Art. 66 (Diverses)

33) Ungarn

18. 8. 1949 (20. 8.): Verfassung der Ungarischen Volksrepublik

StA: - 
34) Vatikan

7. 6. 1929: Legge fondamentale

StA: -

35) Zypern

6. 4. 1960 (16. 8.): (UK) Republic of Cyprus Order in Council, S. I. Nr. 1368

StA: Art. 198 (materiell)

36) Zypern (Nord)

12. 3. 1985: Verfassung der Türkischen Republik Nordzypern

StA: Art. 53 alt (StA durch Gesetz geregelt, Ausbürgerung)

\section{Abhängige Gebiete}

\section{Australien}

Von den 9 Territorien Australiens haben 7 keine StAB (4 Inseln, Antarctis, Nord-Territorien, Canberra), jedoch Christmas und Cocos:

16. 6: 1955 (23. 11.): Cocos (Keeling) Islands Act, Nr. 34

StA: Sec. 14-15 (materiell) Änderungen der StA:

1) 8. 10. 1958, Amd. Act Nr. 67 fügt Sec. 14 (4) an

2) 5. 3. 1979, Amd. Act. Nr. 6, ändert Sec. 14-15, fügt ein Sec. 14 A

2. 9.1958 (1. 10.): Christmas Islands Act, Nr. 41

StA: Sec. 15-16 (materiell)

Änderungen der StA: 17. 12. 1980, Amd. Act 174, ändert Sec. 15-16, fügt ein Sec. 15 A

\section{Dänemark}

Das Autonomiegesetz Grönlands v. 29. 11. 1978 hat keine StAB, aber 23. 3. 1948 Lov Nr. 137 om Faerøernes Hjemmestyre

StA: $\S 10$ (Name in Paß; Faröer sind wohnhafte dän. StA'e; Wahlrecht beschränken auf Faröer)

\section{Finnland}

28. 12. 1951 (1. 1. 52): Gesetz Nr. 670 betr. Autonomie der Aaland-Inseln

StA: Art. 3 (Indigenat, ausführlich)

\section{Frankreich}

4 DOM in Amerika (Guyana, Guadeloupe, Martinique, St. Pierre et Miquélon) und 1 im indischen Ozean (Réunion) sowie zwei Quasi-TOM (Austral-Antarktis, Mayotte) haben keine Verfassungen. Die 3 TOM, die eine Verfassung haben, haben auch eine StAB: StAB:

29. 7. 1961: Loi 814, Statut pour Wallis et Futuna

StA: Art. 2 Satz 1 (Erwerb franz. StA im bisherigen Protektorat) 
28. 12. 1976: Loi 1222; Organisation constitutionelle de la Polynésie Française StA: Art. 7 (Frankreich bleibt zuständig)

12. 7. 1977: Loi 772: Organisation constitutionelle de la Nouvelle Calédonie StA: Art. 62 (dito)

\section{Großbritannien}

Folgende brit. Kolonien haben in ihren Verfassungsregelungen keine StAB: Brit. Antarctis Territory, Brit. Indian Ocean Territory, Cayman Islands, Falkland Islands and Dependencies, Hong Kong, Montserrat, Pitcairn, St. Helena, Turks and Caicos Islands. In den Constitution Orders von 4 Gebieten findet sich auch eine StAB:

14. 2. 1968 (21. 2.): Bermuda Constitution Order, S.I. Nr. 182

StA: Sec. 102 (3) der Constitution (Bermuda Status)

23. 5. 1969 (=): Gibraltar Constitution Order, ohne Nr.

StA: Sec. 17 (3) der Constitution (Gibraltarium Status, Verweisung)

15. 12. 1976 (Jan. 77): Virgin Islands (Constitution) Order, S.I. Nr. 2145

StA: Sec. 2 (2) der Constitution (Belonging to Virgin Islands, ausführlich)

10. 3.1982 (1. 4.): Anguilla Constitution Order, S.I. Nr. 334

StA: Sec. 4 der Order (Citizenship), Sec. 18 (5) der Constitution (Belonging to Anguilla, ausführlich)

\section{Neuseeland}

Für die Ross Dependency (Antarktis) und Tokelau bestehen keine StAB'en, jedoch für Cook und Niue:

17. 11.1964 (4. 8. 65): Cook Islands Constitution Act, Nr. 69

StA: Sec. 6 des Act (das StAG v. NZ v. 1948 galt weiter)

Art. 28 der Constitution (ständige Einwohner), geändert durch Amd. Act. Nr. 2 v. 7. 6. 1965

Art. 46 der Constitution (Gesetz v. NZ nur bei ausdrücklicher Bitte der Inseln auf diese erstreckbar: so StAG v. 1977)

29. 8.1974 (=): Niue Constitution Act, Nr. 42

StA: Sec. 5 des Act (das StAG v. NZ v. 1948 galt weiter)

Art. 17 der Constitution (ständige Einw.)

Art. 36 der Constitution (Ges. v. NZ nur bei ausdrücklicher Bitte der Inseln auf diese erstreckbar; so StAG v. 1977)

\section{Niederlande}

29. 3. $1955(=)$ : Staatsregeling van de Nederlandse Antillen

StA: Art. 6 (StA regelt das Reich)

28. 10. 1954 (29. 12): Statuut voor het Koninkrijk der Nederlanden

StA: Art. 3 (c) (StA ist Reichssache) 


\section{Portugal}

Die einzige portugiesische Kolonie, Macao, hat in ihrem Autonomiegesetz v. 10. 2. 1976 keine StAB

\section{Vereinigte Staaten}

Von den amerikanischen Außenbesitzungen haben keine StAB die Verfassungen von Ost-Samoa, Puerto Rico und den Virgin-Islands und die Ordnung der Panama-Kanalzone. Nur 5 Südseegebiete haben in ihren Verfassungen StAB'en:

1. 8. 1950 (21. 7. ): Guam Organic Act, Ch. 512

StA: Sec. 4 (ändert StAG der USA)

24. 10. 1975 (10. 5. 79): Constitution of the Federated State of Micronesia (= Karolinen) StA: Art. III (Sec. 1-6) (materiell)

5. 12.1976 (9. 1. 78): Constitution of the Northern Marianas

StA: Sec. 8 der Schedule (vorläufige US-Citizens)

21. 12. 1978 (1. 5. 79): Constitution of the Marshall Islands

StA: Art. XI (Sec. 1-3) (materiell)

31. 7. 1980 (1. 1. 81): Constitution of the Republic of Belau (= Palau)

StA: Art. III (Sec. 1-5) (materiell) 